\title{
Do general practices provide equitable access to physical activity interventions?
}

\author{
Sarah L Sowden, Elizabeth Breeze, Julie Barber and Rosalind Raine
}

\begin{abstract}
Background

Exercise referral schemes are widespread across England. National guidance emphasises the need to engage groups that are disadvantaged.

Aim

To examine the influence of socioeconomic deprivation on referral to, and use of, exercise referral schemes.

\section{Design of study}

Cross-sectional analysis of patients referred by general practices to exercise referral schemes between 2004
\end{abstract} and 2006.

\section{Setting}

Six primary care trusts (PCTs) in Greater London.

\section{Method}

Routine data about patients who had been referred to exercise referral schemes were used to estimate risk ratios for referral by general practice deprivation quintile, odds ratios (ORs) for uptake, and ORs for completion of exercise referral schemes by patients' deprivation status quintile.

\section{Results}

All 317 general practices in the six PCTs were included in the referral analysis. Referrals were less likely from general practices serving advantaged socioeconomic areas (adjusted risk ratio for trend across deprivation quintiles $0.84 ; 95 \%$ confidence interval $[\mathrm{Cl}]=0.76$ to 0.93). This study found no association between patients' deprivation status and their likelihood of taking up (adjusted OR, least versus most deprived quintile $1.05 ; 95 \% \mathrm{Cl}=0.83$ to 1.33 ) or completing the scheme (adjusted OR $1.23 ; 95 \% \mathrm{Cl}=0.84$ to 1.79 ).

\section{Conclusion}

General practices within areas of deprivation were more likely to refer patients to exercise referral schemes than practices in more advantaged areas. Once referred, it was found that patients living in areas of deprivation were as likely to take up and to complete the scheme as those living in more advantaged locations. Research is needed to identify the organisational and contextual factors that allow this pattern of service delivery, which appears to facilitate access to care among patients who live in areas of deprivation.

\section{Keywords}

exercise; family practice; health promotion; healthcare disparities; referral and consultation; socioeconomic factors.

\section{INTRODUCTION}

Exercise referral schemes are one of the most widely established primary care physical activity interventions in England (Box 1). ${ }^{1}$ The majority of referrals to these schemes are made by general practices. $^{2}$ National guidance emphasises the importance of ensuring that exercise referral schemes are delivered equitably and explicitly states that they should employ strategies to target and engage people from disadvantaged groups. ${ }^{2}$ Although people from such groups attend primary care more frequently than those from more advantaged socioeconomic groups ${ }^{3}$ (which accords with their greater need for care) they are less likely to use preventative $^{4}$ and specialist $^{5,6}$ health services. This observation, that those in most need of health care are often the least well served in terms of receipt of such care, has been termed the "inverse care law."

The benefits of physical activity for the primary and secondary prevention of a range of clinical conditions ${ }^{8}$ underpin the eligibility criteria for exercise referral schemes. ${ }^{2}$ It is likely that there is higher eligibility for these schemes among patients from groups classified as more socioeconomically

SL Sowden, BA, $M S c, P h D$ research student; $E$ Breeze, $B A$, $M S c, P h D, C S t a t$, DipPopStud, senior lecturer in Health and Social Surveys Research Group; R Raine, BSc, MBBS, MSc, $\mathrm{PhD}, \mathrm{FFPH}$, professor of health services research, Health Care Evaluation Group, Department of Epidemiology and Public Health, University College London; J Barber, BSc, MSc, PhD, lecturer in medical statistics, UCLH/UCL Biomedical Research Unit, London.

Address for correspondence

Sarah L Sowden, Health Care Evaluation Group, Department of Epidemiology and Public Health, UCL, 1-19 Torrington Place, London, WC1E 6BT. E-mail: s.sowden@ucl.ac.uk

Submitted: 21 April 2008; Editor's response: 9 July 2008; final acceptance: 21 August 2008.

(C)British Journal of General Practice.

This is the full-length article of an abridged version published in print. Cite this article as: Br J Gen Pract 2008; DOI: 10.3399/bjgp08X342237. 
deprived because these groups experience more of the conditions for which exercise referral is indicated ${ }^{9}$ and are less likely to engage in leisure-time physical activity $^{8,10}$ than those from groups classified as more socioeconomically advantaged. However, despite higher eligibility for the service, known barriers to participating in physical activity, such as lack of money or access to transport, are socioeconomically patterned. ${ }^{11,12}$ Hillsdon et al demonstrated that the availability of gyms and swimming pools declines with an increase in the level of deprivation such that areas in most need of facilities to assist people to live active lifestyles have fewer resources. ${ }^{10}$

This study examined the association of deprivation with each stage of the exercise referral scheme pathway: from GP referral to uptake through to completion of the scheme. The first objective was to examine the influence of deprivation status of the area within which a general practice was located on the likelihood of referring patients to exercise referral schemes. This provides an indication of the extent to which equity was addressed at the organisational level of general practice, given that this was the main access point for entry to the scheme. The second objective was to examine the influence of patients' deprivation status on the likelihood of attending the initial exercise referral appointment (uptake of the service). The third objective was to examine the influence of patients' deprivation status on the likelihood of attending the final exercise referral appointment (completing the service).

Given that transport is a barrier to engaging in physical activity, ${ }^{11}$ distance both from home and from the referring general practice to the nearest participating exercise referral scheme was considered in this analysis. Furthermore, GP training status has been included as a potential confounder in the analysis. Quality of service is reportedly higher in training practices ${ }^{13}$ and, as such, it is hypothesised that such practices might also have a greater propensity to engage with exercise referral schemes. Training practices are more commonly represented in less deprived areas. ${ }^{13}$

\section{METHOD}

This study used a pragmatic sample of six exercise referral schemes in Greater London, which were able to provide electronic information on each person referred to the scheme over a 24-month period (April 2004 to March 2006) including:

- whether patients attended their initial and final appointments;

- their sociodemographic characteristics;

- information regarding the clinical reason(s) for referral; and

\section{How this fits in}

Exercise referral schemes are one of the most widely established primary care physical activity interventions in England and the majority of referrals to these are made by general practices. National guidance emphasises the need to engage groups that are disadvantaged. General practices within areas of deprivation were more likely to refer patients to exercise referral schemes than practices in more advantaged areas, which suggests referral behaviour may be consistent with efforts to facilitate access to care among patients who live in areas of socioeconomic deprivation. Once referred, patients from more deprived locations were as likely to take up and to complete the scheme as those from more advantaged locations.

- a record of the health professional or health centre that made the referral.

Each exercise referral scheme was located within a primary care trust (PCT) and every general practice within each of these PCTs was able to refer patients to the scheme.

\section{Data sources}

Information came from two sources: the six exercise referral scheme databases of referrals and, for the referral analysis, the family health services database of patients registered with general practices in the participating PCT areas.

Each exercise referral scheme routinely recorded details of those patients who were eligible and had been referred from general practices, as well as their attendance at initial and final exercise referral scheme appointments. Patients' postcodes and general practice postcodes were used to assign the Index of Multiple Deprivation (IMD) $2004^{14}$ at Lower Super Output Area level (LSOA). An LSOA includes an average population of 1500 people. The IMD 2004 scores for each LSOA in England were ranked and these ranks grouped into quintiles, with IMD1 representing the $20 \%$ most deprived LSOAs in

\section{Box 1. Exercise referral schemes.}

Exercise referral schemes (also known as Exercise on Prescription) are a multi-agency intervention involving local primary care trusts, local councils and often voluntary and private leisure service providers. Sedentary patients with existing health problems (such as diabetes, asthma, back pain, and depression) or risk factors for future ill-health (such as risk factors for cardiovascular disease or overweight/obesity) are referred by GPs and other healthcare professionals to a programme of subsidised exercise at a local leisure centre. Similar schemes have been developed in Europe, Australia, New Zealand, and the US. ${ }^{1,24}$ 
England. If patient-level geographical data had have been available, a population-weighted, mean areabased deprivation score could have been calculated for each practice. However, in the absence of these data, an area-based deprivation score linked to the practice postcode was used as an estimate of the practice population's level of socioeconomic deprivation. Practice postcode-linked IMD scores have been shown to provide a valid proxy for a population-weighted measure in the absence of patient-level data. ${ }^{15}$

Data on potential patient-related confounding variables that were available from exercise referral scheme databases included the patient's age, sex, and medical reason(s) for referral. Reasons for referral were grouped into seven binary categories that were consistent across schemes. These consisted of: primary/secondary prevention of cardiovascular disease; diabetes; respiratory conditions; mental health conditions; musculoskeletal/neurological conditions; being overweight/obese; or referral due to old age).

Distances from patients' homes and from referring general practices to the nearest participating exercise referral scheme venues were calculated using postcode coordinates obtained from the National Statistics Postcode Directory. ${ }^{16}$ A further general practice-level covariate, GP training status, was obtained from the GP informatics unit of the London training deanery (personal communication, 2007).

\section{Statistical analyses}

Analysis was performed using STATA SE (version 9.2; College Station, TX, US). Generalised estimating equations (GEEs) were used to estimate risk ratios for referral to exercise referral schemes, odds ratios (ORs) for uptake of exercise referral schemes, and ORs for completion of exercise referral schemes across IMD 2004 quintiles. GEEs were used in preference to standard regression models because they take account of clustering in estimating coefficients and standard errors. It was assumed that patients within a general practice were more similar to each other than to patients from different general practices and hence clustered within general practices. Re-referrals, outof-area referrals, non-general practice referrals, and any records with incomplete data were excluded from the GEE analysis (Figure 1).

For each of the three outcomes in turn, a GEE model with all the covariates that had been identified a priori as potential independent risk factors, or confounders, and the outcome of interest was fitted initially - the full model. A backward elimination procedure was then used to produce three final models that included IMD 2004 and only those covariates that were associated $(P \leq 0.05)$ with the outcome.

\section{Referral analysis}

Information from the family health services database supplied by PCT information managers contained patient population information aggregated into 5-year age/sex groupings. The exposure of interest for the referral analysis was the IMD 2004 quintile of deprivation for each general practice. ${ }^{15}$ The other covariates were age group, sex, scheme area, distance from general practice to nearest participating exercise referral scheme venue, and training status of general practice.

The numerator for estimating referral risks was the total number of patients referred to exercise referral schemes in a given 5-year age/sex/general practice group and the denominator was the total number of patients eligible for exercise referral within the 5-year age/sex/general practice group. The denominator for the referral analysis was derived in the following way. Synthetic estimates of the percentage of the population eligible for exercise referral by age/sex/IMD 2004 quintile were created using information on morbidity and lifestyle characteristics from the Health Survey for England 2004. ${ }^{17}$ Eligibility was defined in terms of inactivity (exercising less than once a week) and also having either one or more of the clinical conditions for which exercise referral is indicated or one or more risk factors for future cardiovascular disease (smoking, family history, body mass index of $\geq 25$ ). The number of patients within each general practice age/sex group who were eligible for exercise referral was then approximated by applying the corresponding age/sex/IMD 2004 quintile synthetic estimate from the Health Survey for England 2004 to the practice population data.

\section{Uptake and completion analyses}

The exposure of interest for both the uptake and completion analyses was the IMD 2004 quintile for each patient (assigned via LSOA of home postcode). The other analysis covariates were age group, sex, scheme area, reason(s) for referral, distance from home to nearest participating exercise referral scheme venue, distance from general practice to nearest exercise referral scheme venue, and training status of general practice.

ORs were calculated, first, with respect to uptake of the service and then for completion of the scheme by those who had attended the first appointment. Area 4 was excluded from this part of the analysis due to incomplete data collection in the uptake and completion fields (Figure 1).

\section{Sensitivity analysis}

GEE models run on the assumption that missing data are missing completely at random. GEE sensitivity analysis was undertaken to assess any influence of 


\section{Exclusions}

Exclusions:

Out-of-area referrals $(n=204)$

Re-referrals ( $n=437$ )

Non-general practice referrals $(n=1781)$

Unknown referral location $(n=334)$
Exercise referral scheme cohort profile

\section{Generalised estimating} equations analysis
GP population profile

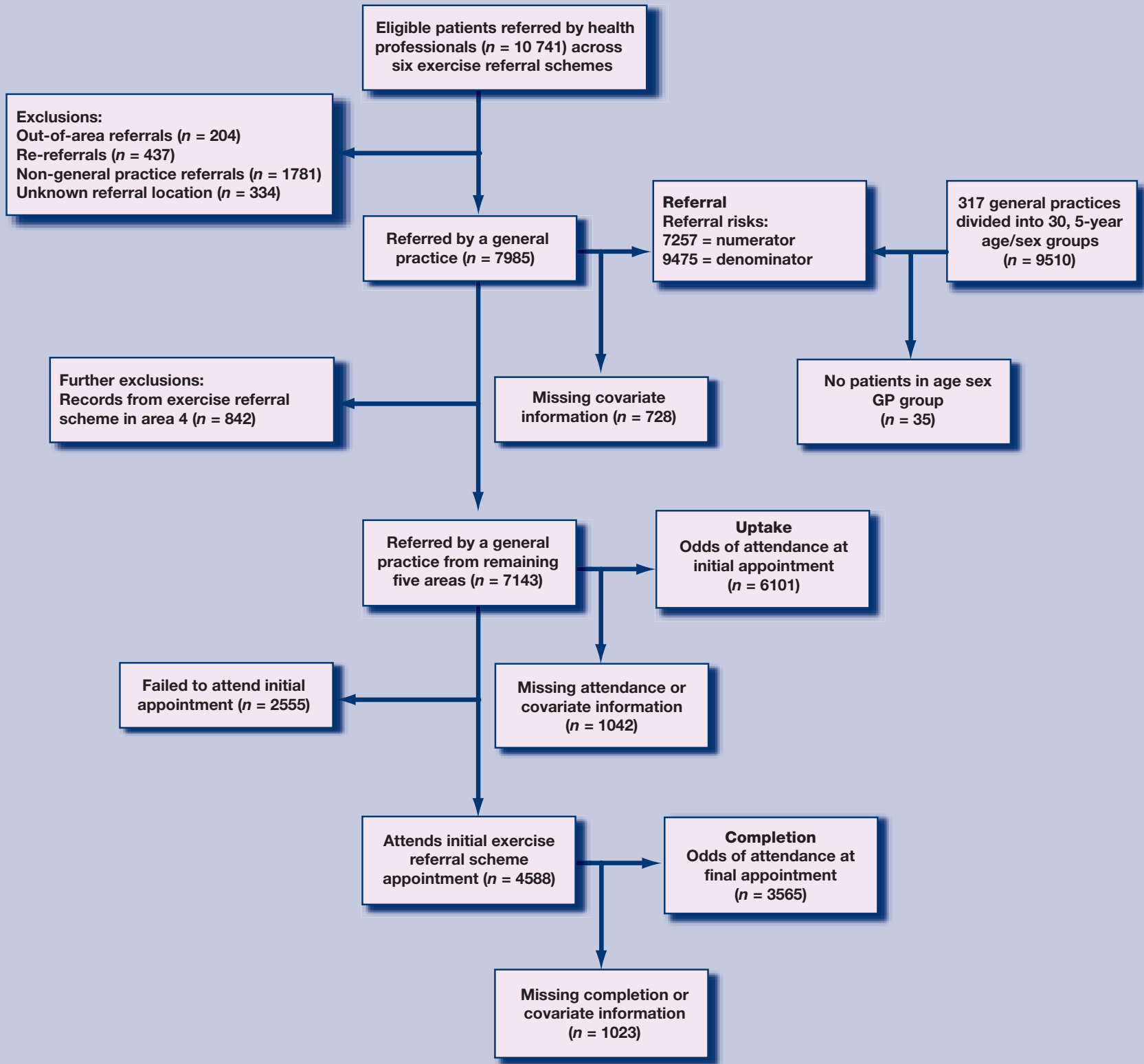

missing outcome data. ${ }^{18}$ (Further details can be obtained directly from the author.)

\section{RESULTS}

The six PCT areas included in the study span the range of deprivation experienced in England. Overall, the study population indicated greater levels of deprivation, on average, than the English population and the exercise referral populations previously studied (Table 1). Consistent with previous studies, referred individuals tended to be of middle age and the majority were female. The proportions of patients taking up and completing exercise referral were low; again, this was consistent with previously reported participation levels (Table 1).

All 317 general practices from the six PCT areas were included in the analysis of referral to exercise referral schemes (Figure 1). After accounting for variations in exercise referral scheme eligibility and controlling for factors found to be associated with referral (age group, sex, scheme area) there was a socioeconomic gradient in referral risk across
Figure 1. Flow diagram illustrating reasons for exclusion of patients from the analysis and numbers of patients examined. 
general practices (Table 2). General practices serving communities that are socioeconomically advantaged were less likely to refer patients to exercise referral schemes compared with those serving communities of deprivation $(P=0.001$, adjusted risk ratio for trend across IMD quintiles $0.84 ; 95 \% \mathrm{Cl}=0.76$ to 0.93 ).

In total, 6101 patients who were referred from the five schemes that reliably monitored scheme activity were included in the uptake analysis (Figure 1). After controlling for factors found to be associated with uptake (age group, sex, being referred for musculoskeletal/neurological conditions), the likelihood of taking up exercise referral schemes was not associated with the socioeconomic status of patients who had been referred (adjusted $P=0.85$, test for trend $P=0.89$ ).

Some 3565 patients who took up the service were included in the completion analysis (Figure 1). After controlling for factors found to be associated with completion (age group, scheme area, being referred for prevention of cardiovascular disease and/or diabetes, training status of general practice) the likelihood of completing exercise referral schemes was not found to be associated with the socioeconomic status of referred patients (adjusted $P=0.06$, test for trend $P=0.20$; Table 2).

In total, 39 and 537 patients had missing information on attendance at the first and last exercise referral scheme appointment respectively; as a result they were excluded from the relevant GEE analysis. The sensitivity analysis undertaken to explore the influence of missing outcome information (not shown) did not alter the substantive results for IMD 2004.

\section{DISCUSSION}

\section{Summary of main findings}

The main findings show that general practices within areas of deprivation were more likely to refer to exercise referral schemes than their counterparts in more advantaged areas. This was the case even after accounting for the greater levels of eligibility for exercise referral schemes present within those communities that are classified as deprived. This study's findings suggest that exercise referral schemes do not comply with the inverse care law, which is commonly reported for preventative health services. ${ }^{4}$

Once given access to the system, patients living in areas of deprivation were as likely as those from more socioeconomically advantaged areas to take up and complete the scheme. This suggests that concerns that leisure-centre based schemes are unlikely to recruit people from groups that are classified as deprived, and that such people are unlikely to adhere to exercise programmes, may be unfounded. ${ }^{19,20}$ However, optimism must be tempered by the fact that overall participation levels were low and the long-term effectiveness and cost effectiveness of exercise referral schemes has yet to be established. ${ }^{21}$

\section{Strengths and limitations of the study}

This is the first study to examine the likelihood of referral to exercise referral schemes, enabling the association between deprivation and service use to be studied across the whole exercise referral scheme pathway. This has been achieved using an innovative approach to quantify eligibility for exercise referral across general practice populations.

Table 1. Characteristics of patients referred compared with previous exercise referral scheme studies.

\begin{tabular}{|c|c|c|c|c|c|}
\hline & $\begin{array}{l}\text { This research dataset } \\
\text { (combined across six } \\
\text { participating schemes) }\end{array}$ & $\begin{array}{l}2005 \text { systematic review of } \\
\text { exercise referral schemes }{ }^{28} \\
\text { (included four RCTs and } \\
\text { five non-RCT evaluations) }\end{array}$ & $\begin{array}{l}2008 \text { systematic review of } \\
\text { exercise referral schemes } \\
\text { (included six RCTs and } \\
11 \text { non-RCT evaluations) }\end{array}$ & $\begin{array}{c}2005 \\
\text { evaluation }^{24}\end{array}$ & $\begin{array}{c}2007 \\
\text { evaluation }^{25,29}\end{array}$ \\
\hline Uptake, \% & $66^{a}$ & $23-79$ & $26-100$ & 79 & 65 \\
\hline Completion, \% & $39^{b}$ & $12-56$ & $12-42$ & Not reported & 31 \\
\hline Mean IMD 2004 score $^{c}$ & 28.8 & Not reported & Not reported & Not reported & 16.5 \\
\hline Mean age, years & 51 & Middle aged and older & Not reported & 51 & 51 \\
\hline Females, \% & 65 & Around 60 & Not reported & 61 & 61 \\
\hline
\end{tabular}

${ }^{a} 66 \%$ is $\%$ of uptake for those with no missing covariate data $(\mathrm{n}=6101) .{ }^{b} 39 \%$ is $\%$ completion for those with no missing covariate data $(\mathrm{n}=3565) .{ }^{c}$ Higher score $=$ more deprived. ${ }^{~} I M D 1=20 \%$ most deprived areas of England. ${ }^{\circ}$ Of 7985 patients referred by general practice (Figure 1), those with IMD recorded ( $\mathrm{n}=7612$ ). ${ }^{f}$ Data from Table 4 in Harrison et al $2005,{ }^{24}$ patients referred with IMD and referral condition recorded ( $\mathrm{n}=5237$ ). IMD $=$ Index of Multiple Deprivation $R C T=$ randomised controlled trial. 
In contrast with previous primary research examining factors associated with use of exercise referral schemes that have focused on single schemes, we examined six exercise referral schemes across Greater London. Comparisons with previously published studies show that the demographic profile and participation levels of those patients referred in this research are typical of other schemes running across England (Table 1). Therefore, the current research is likely to be generalisable to other exercise referral schemes, especially those that are located in socially-diverse urban populations.
A further strength is that a wide range of theoretically plausible variables that might confound any relationship between socioeconomic status and service use were taken into account, including several that have not been examined before in this context (distance from home or general practice to nearest participating exercise referral scheme venue, training status of the general practice).

Robust statistical techniques were used to take account of clustering of referrals within general practices. These techniques were not applied in previous examinations of exercise referral schemes.

\section{Table 2. Final 3 models showing the association between Index of Mulitple Deprivation (IMD) 2004 and referral $^{\mathrm{a}}$ uptake $^{\mathrm{b}}$ and completion ${ }^{\mathrm{b}}$ of exercise referral schemes. ${ }^{\mathrm{c}}$}

\begin{tabular}{|c|c|c|c|c|c|c|c|c|c|}
\hline \multirow[b]{2}{*}{ Exposure variable } & \multicolumn{3}{|c|}{$\begin{array}{l}\text { Risk ratios for referral to } \\
\text { exercise referral schemes }\end{array}$} & \multicolumn{3}{|c|}{$\begin{array}{l}\text { ORs for uptake } \\
\text { of exercise referral schemes }\end{array}$} & \multicolumn{3}{|c|}{$\begin{array}{l}\text { ORs for completion of } \\
\text { exercise referral schemes }\end{array}$} \\
\hline & $n=9475$ & Risk ratio & $95 \% \mathrm{Cl}$ & $n=6101$ & OR & $95 \% \mathrm{Cl}$ & $n=3565$ & OR & $95 \% \mathrm{Cl}$ \\
\hline IMD quintile & $P=0.004$ & & & $P=0.85$ & & & $P=0.060$ & & \\
\hline 1 (most deprived) & 4415 & 1 & & 2335 & 1 & & 1422 & 1 & \\
\hline 2 & 2364 & 0.75 & 0.53 to 1.05 & 1595 & 1.05 & 0.93 to 1.21 & 881 & 0.89 & 0.71 to 1.11 \\
\hline 3 & 1286 & 0.62 & 0.40 to 0.95 & 786 & 0.94 & 0.77 to 1.15 & 395 & 0.92 & 0.63 to 1.34 \\
\hline 4 & 420 & 0.42 & 0.24 to 0.72 & 575 & 0.99 & 0.78 to 1.25 & 348 & 1.47 & 0.96 to 2.24 \\
\hline \multirow[t]{2}{*}{5 (least deprived) } & 990 & 0.53 & 0.37 to 0.76 & 810 & 1.05 & 0.83 to 1.33 & 519 & 1.23 & 0.84 to 1.79 \\
\hline & \multicolumn{3}{|c|}{$P=0.001$ Test for trend } & $P=0.89$ & \multicolumn{2}{|c|}{ Test for trend } & $P=0.20$ & \multicolumn{2}{|c|}{ Test for trend } \\
\hline $\begin{array}{l}\text { Overall (trend for one } \\
\text { quintile increase in IMD) }\end{array}$ & 9475 & 0.84 & 0.76 to 0.93 & 6101 & 1.00 & 0.95 to 1.06 & 3565 & 1.06 & 0.97 to 1.17 \\
\hline Age group & $P<0.001$ & & & $P<0.001$ & & & $P<0.001$ & & \\
\hline $16-29$ years & 1902 & 1 & & 556 & 1 & & 231 & 1 & \\
\hline 30-44 years & 1902 & 2.30 & 2.01 to 2.62 & 1624 & 1.67 & 1.34 to 2.08 & 866 & 1.30 & 0.96 to 1.77 \\
\hline $45-59$ years & 1902 & 2.79 & 2.39 to 3.26 & 2110 & 2.09 & 1.68 to 2.61 & 1277 & 1.77 & 1.27 to 2.46 \\
\hline $60-74$ years & 1899 & 2.21 & 1.87 to 2.63 & 1550 & 2.67 & 2.14 to 3.33 & 1027 & 2.91 & 2.04 to 4.16 \\
\hline$\geq 75$ years & 1870 & 0.34 & 0.25 to 0.48 & 261 & 2.43 & 1.70 to 3.46 & 164 & 2.71 & 1.65 to 4.46 \\
\hline Sex & $P<0.001$ & & & $P<0.001$ & & & $\mathrm{NFM}^{+}$ & & \\
\hline Male & 4737 & 1 & & 1778 & 1 & & & & \\
\hline Female & 4738 & 2.74 & 2.52 to 2.96 & 4323 & 1.33 & 1.18 to 1.49 & & & \\
\hline Scheme area & $P<0.001$ & & & $\mathrm{NFM}^{+}$ & & & $P<0.001$ & & \\
\hline Area 1 & 2002 & 1 & & & & & 509 & 1 & \\
\hline Area 2 & 1590 & 2.18 & 1.57 to 3.02 & & & & 1319 & 0.43 & 0.32 to 0.58 \\
\hline Area 3 & 1938 & 1.16 & 0.81 to 1.64 & & & & 1194 & 4.45 & 3.28 to 6.03 \\
\hline Area 4 & 1196 & 0.68 & 0.45 to 1.03 & & & & $\mathrm{Nl}^{e}$ & & \\
\hline Area 5 & 1279 & 0.06 & 0.01 to 0.42 & & & & 317 & 13.49 & 8.78 to 20.72 \\
\hline Area 6 & 1470 & 0.19 & 0.13 to 0.28 & & & & 226 & 0.45 & 0.29 to 0.70 \\
\hline $\begin{array}{l}\text { Referred for musculoskeletal/ } \\
\text { neurological reasons }\end{array}$ & $/ N T^{d}$ & & & $P=0.036$ & & & $\mathrm{NFM}^{+}$ & & \\
\hline No & & & & 4562 & 1 & & & & \\
\hline Yes & & & & 1539 & 1.18 & 1.01 to 1.38 & & & \\
\hline Referred for diabetes & $N T^{d}$ & & & $\mathrm{NFM}^{+}$ & & & $P<0.007$ & & \\
\hline No & & & & & & & 2967 & 1 & \\
\hline Yes & & & & & & & 598 & 0.76 & 0.63 to 0.93 \\
\hline $\begin{array}{l}\text { Referred for primary or } \\
\text { secondary CVD prevention }\end{array}$ & $N T^{d}$ & & & $\mathrm{NFM}^{+}$ & & & $P=0.020$ & & \\
\hline No & & & & & & & 1973 & 1 & \\
\hline Yes & & & & & & & 1592 & 1.22 & 1.03 to 1.45 \\
\hline Training general practice & $\mathrm{NFM}^{+}$ & & & $\mathrm{NFM}^{+}$ & & & $P=0.048$ & & \\
\hline No & & & & & & & 2224 & 1 & \\
\hline Yes & & & & & & & 1341 & 0.81 & 0.65 to 1.00 \\
\hline
\end{tabular}

aIMD quintile of general practice. ${ }^{b} I M D$ quintile of patient's home. ${ }^{c}$ Other factors associated with outcome $(\mathrm{P} \leq 0.05)$ and which are therefore included in the final model also reported. ${ }^{d}$ These variables were unavailable for the referral analysis. ${ }^{\circ}$ Area 4 data excluded from analysis. ${ }^{f}$ Variables did not improve fit of the model. $C V D=$ cardiovascular disease. $N F M=$ not in final model. $N I=$ not included. $N T=$ not tested. $\mathrm{OR}=$ odds ratio. 
There were also some limitations to this study. Only six of the 30 exercise referral schemes established across Greater London were examined. This was because no standard minimum dataset has been developed for exercise referral schemes and, as a result, only seven schemes collected sociodemographic information and monitored uptake and completion of those patients who had been referred; six of these schemes agreed to participate in the research.

This analysis took account of eligibility for exercise referral because it was not possible to examine referral, uptake, and completion having taken account of clinical need for the scheme. An assessment of need for exercise referral depends on having evidence of the effectiveness of the intervention (that is, the ability to benefit) for different conditions and risk factors. National Institute for Health and Clinical Excellence (NICE) guidance has recently highlighted the lack of such evidence and recommended that commissioners should only endorse schemes that are part of controlled studies to determine effectiveness. ${ }^{22,23}$ Such research requires the collection of standard data on health outcomes. It was not possible to examine associations between use of exercise referral schemes and health outcomes because of the heterogeneity of outcome measures currently collected by schemes. This was coupled with poor reliability among some exercise referral schemes in terms of their recording of patients' post-intervention health status.

It would have been valuable to have examined referral at the patient level in addition to referral at the general practice level. However, under current ethical guidance this would have required consent from each patient in all 317 practices across the six PCTs to enable patient records to be matched with exercise referral scheme referrals. This was not practical. Uptake and completion were examined at the patient level using an area-level indicator of deprivation because individual-level data on patients' socioeconomic status (such as employment or educational attainment) were not recorded in the databases examined.

Area-level indicators of deprivation based on postcodes are commonly used in health services research. ${ }^{15,24,25}$ It is recognised that if individuals do not conform to the socioeconomic profile of their residential area, or if the location of the general practice does not accurately reflect the profile of its patients, there may be over- or under-estimation of the effect being examined. ${ }^{26}$ To minimise the divergence between individual and area characteristics the smallest area level (the LSOA) for which IMD 2004 is available was selected. IMD 2004 was used in this study because it draws on a range of sources, including data from the census, from administrative databases detailing claimants of income and disability benefits, hospital episodes statistics, educational attainment records, and Home Office crime statistics. ${ }^{14}$ As such, it adopts a holistic conceptualisation of deprivation; it is also updated every 3 years, unlike other measures, such as the Townsend and Carstairs indices. ${ }^{27}$

Finally, 39 patients were excluded from the uptake analysis and 537 patients from the completion analysis due to missing outcome data (Figure 1). If missing data are not missing at random but are systematically distributed within the dataset this could bias the results. However, sensitivity analysis undertaken for outcome data not missing at random using $\mathrm{GEE}^{18}$ did not alter the substantive results for the association between deprivation and uptake or completion.

\section{Comparison with existing literature}

Previous research is limited to analyses of single schemes and has produced conflicting results. In common with the current study's findings, a study of an exercise referral scheme in an urban area in the northwest of England found that patient deprivation status had no influence on the likelihood of attending the first exercise referral scheme appointment ${ }^{24}$ and a study of a scheme in rural Somerset found that patients from areas of deprivation were just as likely to complete the scheme as their more advantaged counterparts. ${ }^{25}$ However, in contrast to this study's results, patients in areas of deprivation in Somerset were less likely to take up their initial appointment. ${ }^{25}$

\section{Implications and future research}

This study demonstrated that general practices within areas of deprivation were more likely to refer patients to exercise referral schemes than practices in more advantaged areas, even after accounting for the greater levels of eligibility for exercise referral within communities classified as deprived. Research is now needed to identify the organisational and contextual factors that enable this pattern of service delivery that appears to facilitate access to care among patients from areas of deprivation and, effectively, reverses the inverse care law. The success of these schemes in promoting physical activity among groups from areas of deprivation also underlines the need to evaluate their effectiveness in improving health and in reducing health inequalities, as recommended by NICE. ${ }^{23}$

\section{Funding body}

The research was funded by an ESRC/MRC PhD studentship Ethical approval

Prior ethical approval was granted by Committee A of the Joint UCL/UCLH Committees on the Ethics of Human Research (06/Q0505/65). Research governance approval was obtained from the six primary care trusts participating in the research 


\section{Competing interests}

The authors have stated that there are none

\section{Acknowledgements}

We thank the exercise referral scheme managers, scheme personnel, and those working within Public Health in the primary care trusts in the six areas within Greater London who participated in this research.

\section{Discuss this article}

Contribute and read comments about this article on the Discussion Forum: http://www.rcgp.org.uk/bjgp-discuss

\section{REFERENCES}

1. Sowden SL, Raine R. Running along parallel lines: how political reality impedes the evaluation of public health interventions. A case study of exercise referral schemes in England. J Epidemiol Community Health 2008; 62(9): 835-841.

2. Department of Health. Exercise referral systems: a national quality assurance framework. London: Department of Health, 2001. http://www.dh.gov.uk/prod_consum_dh/groups/dh_digitalassets/ @dh/@en/documents/digitalasset/dh_4079009.pdf (accessed 27 Aug 2008).

3. Morris S, Sutton M, Gravelle H. Inequity and inequality in the use of health care in England: an empirical investigation. Soc Sci Med 2005; 60(6): 1251-1266.

4. Goddard M, Smith P. Equity of access to health care services: theory and evidence from the UK. Soc Sci Med 2001; 53(9): 1149-1162.

5. Dixon A, Le Grand J, Henderson J, et al. Is the British National Health Service equitable? The evidence on socioeconomic differences in utilization. J Health Serv Res Policy 2008; 12(2): 104-109.

6. Hutchings A, Raine R, Brady A, et al. Socioeconomic status and outcome from intensive care in England and Wales. Med Care 2004 42(10): 943-951.

7. Hart JT. The inverse care law. Lancet 1971; 1(7696): 405-412.

8. Department of Health, Physical Activity, Health Improvement and Prevention. At least five a week: evidence on the impact of physical activity and its relationship to health. A report from the Chief Medical Officer. London: Department of Health, 2004. http://www.dh.gov.uk/prod_consum_dh/groups/dh_digitalassets/@ dh/@en/documents/digitalasset/dh_4080981.pdf (accessed 26 Aug 2008).

9. Marmot M, Wilkinson RG. Social determinants of health. 2nd edn. Oxford: Oxford University Press, 2006.

10. Hillsdon M, Panter J, Foster C, Jones A. Equitable access to exercise facilities. Am J Prev Med 2007; 32(6): 506-508.

11. Chinn DJ, White M, Harland J, et al. Barriers to physical activity and socioeconomic position: implications for health promotion I Epidemiol Community Health 1999; 53(3): 191-192.

12. Tai SS, Gould M, Smith P, Iliffe S. Promoting physical activity in general practices: should prescribed exercise be free? J R Soc Med 1999; 92(2): 65-67.

13. Ashworth $\mathrm{M}$, Seed $\mathrm{P}$, Armstrong $\mathrm{D}$, et al. The relationship between social deprivation and the quality of primary care: a national survey using indicators from the UK Quality and Outcomes Framework. Br J Gen Pract 2007; 57(539): 441-448.

14. Noble M, Wright G, Dibben C, et al. The English indices of deprivation 2004 (revised). London: The Stationery Office, 2003.

15. Strong M, Maheswaran R, Pearson T. A comparison of methods for calculating general practice level socioeconomic deprivation. Int $J$ Health Geogr 2006; 5: 29.

16. Office for National Statistics. National Statistics Postcode Directory. London: Office for National Statistics, 2006.

17. National Centre for Social Research, Department of Epidemiolog and Public Health University College London. Health Survey for England 2004. http://www.data-archive.ac.uk/findingData/ snDescription.asp?sn=5439 (accessed 3rd Sept 2008).

18. Carpenter J, Kenward M. Guidelines for handling missing data in Social Science Research.

http://www.lshtm.ac.uk/msu/missingdata/guidelines.pdf (accessed 27 Aug 2008).

19. Sallis JF, Hovell MF. Determinants of exercise behaviour. Exerc Sport Sci Rev 1990; 18: 307-330.

20. Hillsdon M, Thorogood M. A systematic review of physical activity promotion strategies. Br J Sports Med 1996; 30(2): 84-89.

21. Williams NH, Hendry M, France B, et al. Effectiveness of exercisereferral schemes to promote physical activity in adults: systematic review. Br J Gen Pract 2007; 57(545): 979-986.

22. National Public Health Collaborating Centre. A rapid review of the effectiveness of exercise referral schemes to promote physical activity in adults. London: NICE, 2006

23. National Institute for Health and Clinical Excellence. Four commonly used methods to increase physical activity: brief interventions in primary care, exercise referral schemes, pedometers and community-based exercise programmes for walking and cycling. Public Health Intervention Guidance No 2. London: NICE 2006. http://www.nice.org.uk/nicemedia/pdf/PH002_physical_ activity.pdf (accessed 27 Aug 2008)

24. Harrison RA, McNair F, Dugdill L. Access to exercise referral schemes - a population based analysis. J Public Health (Oxf) 2005; 27(4): 326-330.

25. Gidlow C, Johnston LH, Crone D, et al. Socio-demographic patterning of referral, uptake and attendance in Physical Activity Referral Schemes. J Public Health (Oxf) 2007; 29(2): 107-113.

26. Galobardes B, Shaw M, Lawlor DA, et al. Indicators of socioeconomic position (part 2). J Epidemiol Community Health 2006; 60(2): 95-101.

27. Shaw M, Galobardes B, Lawlor DA, et al. The handbook of inequality and socioeconomic position: concepts and measures. Bristol: The Policy Press, 2007.

28. Gidlow C, Johnston LH, Crone D, James D. Attendance of exercise referral schemes in the UK: a systematic review. Health Educ J 2005; 64(2): 168-186.

29. James DV, Johnston LH, Crone D, et al. Factors associated with physical activity referral uptake and participation. J Sports Sci 2008; 26(2): 217-224. 